\title{
Ostéochimionécrose extensive de la mandibule chez un patient présentant une ostéomalacie et traité par dénosumab pour une tumeur osseuse sphénoïdale
}

\author{
Anne-Gaëlle Bodard ${ }^{1,2}$, Aline Desoutter ${ }^{1}$, Samuel Salino ${ }^{1}$ \\ 1 Centre Léon Bérard, 28 rue Laennec 69008 Lyon, France \\ 2 Faculté d'odontologie, 11 rue Guillaume Paradin, 69008 Lyon, France
}

(Reçu le 19 mai 2015, accepté le 23 juin 2015)

Mots clés : ostéonécrose / dénosumab / ostéomalacie

Key words:

osteonecrosis / denosumab / osteomalacia

\begin{abstract}
Résumé - Observation : Le cas d'un patient âgé de 68 ans, traité par dénosumab, calcitriol et phosphates pour une ostéomalacie et une tumeur à cellules géantes du sphénoïde est rapporté. Le signe clinique inaugural était une douleur sourde au niveau de la mandibule. L'orthopantomographie montrait un double contour au niveau de l'ensemble de la mandibule, et la scintigraphie une hyperfixation de toute la mandibule. Après plusieurs mois de polyantibiothérapie et une tentative d'arrêt du dénosumab, de larges plages d'exposition osseuse étaient notées au niveau de tout le versant alvéolaire lingual de la mandibule. La limitation d'ouverture buccale a été brutalement suivie d'un blocage de la fermeture buccale par fracture comminutive des branches montantes mandibulaires. Discussion : L'ostéochimionécrose, souvent imputable aux bisphosphonates, peut également être associée à la prise de dénosumab. Le dénosumab est un anticorps monoclonal anti RANK-ligand ciblant les ostéoclastes. L'étendue de l'ostéonécrose est probablement liée à l'existence d'une altération du remodelage osseux (ostéomalacie). L'absence d'alternative thérapeutique dans le cas clinique présenté et les bénéfices cliniques constatés doivent faire préférer un traitement antirésorptif malgré un risque accru de développer une ostéochimionécrose.
\end{abstract}

\begin{abstract}
Extensive osteochemonecrosis of the mandible in a patient treated with denosumab for a sphenoidal osseous tumor. Description: The case of a 68-year-old patient, treated with denosumab, calcitriol and phosphates for osteomalacia and a giant cell tumor of the sphenoid, is reported. The initial symptom was dull mandibular pain. The radiological signs were a periosteal reaction of the whole mandible in the orthopantomography, and hyperfixation of the mandible in the scintigraphy. After various types of polyantibiotic therapy, and despite an interruption of the denosumab, large amounts of exposed bone were visible on the lingual side of the corpus mandibulae. A comminuted fracture of both condylar processes was also noted. Discussion: Both bisphosphonates and denosumab can induce osteochemonecrosis. Denosumab is an anti-RANK ligand antibody targeting osteoclasts. The large extension of osteonecrosis can be related to concomitant alterations of bone remodeling (osteomalacia). The lack of therapeutic alternatives in this case and the clinical benefits indicate antiresorptive treatment, despite an increased risk of osteochemonecrosis.
\end{abstract}

\section{Observation clinique}

Un patient traité depuis six ans par calcitriol et phosphates pour une ostéomalacie s'est présenté en consultation suite à la découverte d'une tumeur à cellules géantes du sphénoïde et du sinus caverneux gauche. Un ptosis de la paupière gauche avec diminution de la vision et une paralysie du tronc côté gauche ont amené à la réalisation d'examens complémentaires d'imagerie médicale et d'une biopsie révélant une tumeur à cellules géantes anévrismatique sphénoïdale, non résécable. La radiothérapie ayant été réfutée, quinze perfusions mensuelles de dénosumab (119 mg) ont été proposées. Après 6 perfusions, le patient présentait une stabilité lésionnelle, voire une légère régression, un rétablissement de la paupière, une récupération de la vision de l'œil gauche. Après 7 perfusions, le patient se plaignait de douleurs sourdes, bilatérales, de la mandibule, d'une hypoesthésie labio-mentonnière bilatérale et d'une limitation de l'ouverture buccale. Le patient 


\section{A S C L I N I Q U E}

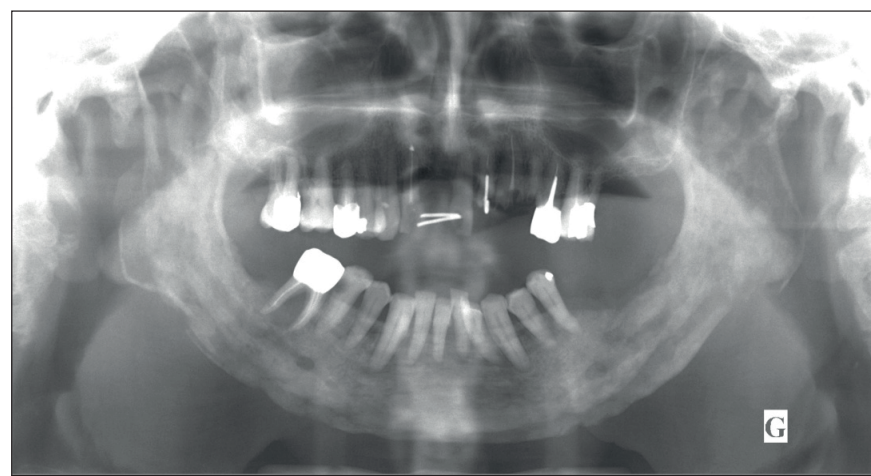

Fig. 1. Orthopantomographie à 17 mois de traitement : noter le double contour et la dystrophie osseuse extensive.

Fig. 1. Orthopantomography after 17 months of denosumab: note the double-spaced contour and the extensive bone dystrophy.

scorait la douleur à 4 sur une échelle de 0 à 10 , avec des pics à 8-9. La scintigraphie réalisée à ce stade a montré une hyperfixation de l'ensemble de la mandibule. Un scanner a objectivé une réaction périostée de l'ensemble du corps mandibulaire, révélée par une image en double contour. Cliniquement, il n'existait aucun signe d'ostéonécrose à ce stade. Un traitement par antalgiques de palier 3 (sulfate de morphine) a été instauré, permettant une diminution des douleurs au bout de 2 à 3 mois à des scores maximaux de 3 à 4 (échelle EVA). Dix mois plus tard (soit à 17 mois de traitement), le patient s'est à nouveau présenté à la consultation avec une infection mandibulaire bilatérale et des expositions osseuses multiples ( $4 \times 3 \mathrm{~mm}$ à droite et à gauche) au niveau du versant lingual du corpus mandibulaire. L'orthopantomographie (Fig. 1) a révélé une image en double contour liée à une réaction périostée, une perte des trabéculations osseuses et une inhomogénéité de l'ensemble de la mandibule. Un traitement symptomatique a été instauré : antibiothérapie probabiliste à large spectre (association d'amoxicilline et de spiramycine), antalgiques et fraisage des spicules irritants pour la muqueuse et la langue. Après concertation multidisciplinaire, il a été convenu d'interrompre le dénosumab. Au bout de 11 mois, celui-ci a dû être repris du fait de l'évolution tumorale. La lésion était en effet passée de $16 \times 22 \times 11 \mathrm{~mm}$ au niveau du corps sphénoïdal à $20 \times 25 \times 24 \mathrm{~mm}$, envahissant la paroi du sinus caverneux. Trois mois après sa réintroduction, le patient est revenu consulter en urgence pour impossibilité de fermeture buccale. Un large séquestre $(90 \times 18 \mathrm{~mm})$ non mobile était visible sur le versant lingual mandibulaire, et le scanner effectué a montré une fracture comminutive de l'ensemble du corps, des condyles et des branches montantes mandibulaires (Fig. 2). La douleur et l'infection ont été contrôlées par polyantibiothérapie. Un diagnostic d'infection aspergillaire a été obtenu, associé à des germes multiples dont certains résistants (Escherichia coli, Staphylococcus epidermitis) pour lesquels un traitement antibiotique à large spectre a été prescrit et poursuivi au long cours : itraconazole, pristinamycine et ertapénème.

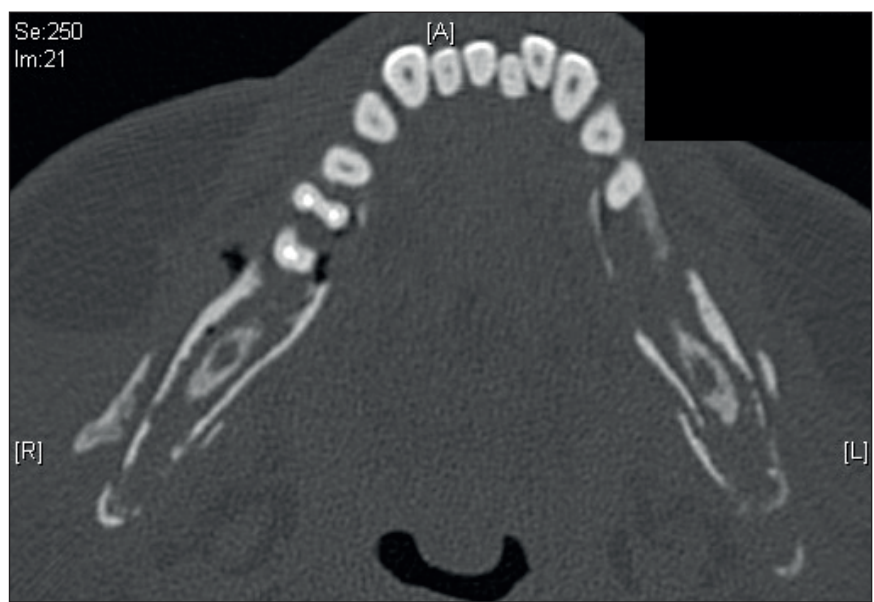

Fig. 2. Scanner objectivant l'atteinte toto-mandibulaire. Fig. 2. CT showing involvement of the whole mandible.

Une surveillance clinique tous les 2 mois, radiologique tous les 6 mois, avec fraisage des spicules osseux traumatiques, a été mise en place.

\section{Discussion}

Ces dernières décennies ont vu l'émergence d'une pathologie iatrogène, l'ostéochimionécrose, imputable aux bisphosphonates et à certaines thérapies ciblées. Le dénosumab est un anticorps monoclonal humain spécifique du RANK ligand. En se fixant sur le ligand, il empêche l'activation du récepteur RANK des ostéoclastes et de leurs précurseurs, inhibant ainsi la différenciation, la fonction et la survie ostéoclastique. La résorption osseuse est ainsi diminuée. L'ostéonécrose imputable au dénosumab (DONJ) est similaire à l'ostéonécrose imputable aux bisphosphonates (BRONJ) [1]. Leur traitement est encore difficile et la prévention reste aujourd'hui la meilleure solution thérapeutique.

Les tumeurs osseuses à cellules géantes sont considérées par certains comme bénignes, par d'autres comme malignes. Leur traitement repose principalement sur la chirurgie et la radiothérapie. Le dénosumab trouve son indication lorsque la chirurgie ne peut être réalisée ou en préparation de l'intervention [2]. La localisation (proximité avec les structures cérébrales, envahissement de la paroi du sinus caverneux) et l'inopérabilité de la tumeur du cas présenté rendait la proposition d'administration de dénosumab pertinente.

De nombreuses études comparent le dénosumab et les bisphosphonates. Pour certains auteurs [3], le dénosumab aurait une activité inhibitrice des ostéoclastes plus importante que les bisphosphonates, avec de plus faibles effets secondaires à long terme du fait d'une non-incorporation osseuse. Sa demivie sérique est courte, de 25 à 29 jours. Toutefois, ce médicament est reconnu pour entraîner des ostéonécroses. Dans une étude de phase III, Fizazi [4] retrouve une incidence des 
ONM induites par le dénosumab (DONJ) de $2 \%$ versus $1 \%$ pour le zolédronate (BRONJ). Les signes radiologiques sont semblables entre les deux entités, avec des images de double contour sur l'orthopantomographie et le scanner, liées à une réaction périostée. Dans le cas présenté, l'importance de la DONJ et les signes radiologiques extensifs sont probablement dus à la fois au traitement par phosphates, à l'inflammation causée par l'accumulation de tissu ostéoïde due à l'ostéomalacie ainsi qu'au traitement par dénosumab. L'ostéomalacie est caractérisée par une accumulation anormale de tissu ostéoïde ; dans sa forme hypophosphatémique, il existe un déficit de métabolisme phosphocalcique, traité par calcitriol. Le traitement vise ainsi à augmenter la résorption ostéoclastique, provoquant un relargage calcique. L'administration concomitante de phosphates permet un rééquilibrage de cette balance.

Les premiers symptômes oraux sont apparus précocement, soit 7 mois après l'introduction du dénosumab. Ils étaient principalement liés à l'inflammation, puisque dans un second temps les douleurs ont diminué concomitamment à l'apparition de plages de dénudation osseuse. Les délais habituels de survenue d'une DONJ oscillent entre 8 et 13 mois de traitement $[3,5]$. La fracture spontanée, comminutive, extensive pourrait être expliquée par l'utilisation concomitante d'un agent antirésorptif et de taux sériques bas de phosphatase alcaline. Un cas clinique de fracture fémorale atypique a été rapporté chez une patiente hypophosphatasique traitée par bisphosphonates [6].

La littérature semble affirmer que les DONJ pourraient être résolutives plus rapidement que les BRONJ du fait de la demivie courte du dénosumab. Dans le cas présenté, on pourrait s'interroger sur le rôle de la dérégulation du métabolisme phosphocalcique dans la survenue de cette DONJ toto-mandibulaire. En effet, une étude a montré que des patients porteurs d'une ostéomalacie présentaient plus fréquemment des BRONJ. L'ostéomalacie serait donc un facteur de risque de survenue de l'ostéochimionécrose [7].

Malgré un suivi clinique régulier et une adaptation thérapeutique, le patient a développé une ostéonécrose toto-mandibulaire. Il semble donc appartenir à une catégorie à haut risque d'ostéonécrose du fait d'effets agonistes du dénosumab sur son métabolisme osseux préalablement altéré par l'ostéomalacie. Ces facteurs de risque soulèvent la question de la pertinence de l'instauration d'un traitement par antirésorptif chez des patients présentant une altération métabolique osseuse. Toutefois, dans le cas clinique présenté, l'absence de solution thérapeutique et les bénéfices cliniques constatés rendent cette solution thérapeutique incontournable. L'augmentation présumée du risque de développer une DONJ de plus grande étendue doit toutefois être considérée dans la balance bénéfice/risque lors du choix thérapeutique. Une adaptation de l'information quant aux effets secondaires délivrée aux patients doit être mise en place, ainsi qu'une surveillance clinique régulière.

\section{Conflits d'intérêt : aucun}

\section{Références}

1. Malan J, Ettinger K, Naumann E, Beirne OR. The relationship of denosumab pharmacology and osteonecrosis of the jaws. Oral Surg Oral Med Oral Pathol Oral Radiol 2012;114(6):671-6.

2. Van der Heijden L, Dijkstra PD, van de Sande MA, Kroep JR, Nout RA, Van Rijswijk CS, et al. The clinical approach toward giant cell tumor of bone. Oncologist 2014;19(5):550-61.

3. Pichardo SEC, Kuypers SCC, Van Merkesteyn JPR. Denosumab osteonecrosis of the mandible: a new entity? A case report. J Craniomaxillofac Surg 2013;41(4):e65-9.

4. Fizazi K, Carducci M, Smith M, Damiao R, Brown J, Karsch $L$, et al. Denosumab versus zoledronic acid for treatment of bone metastases in men with castration-resistant prostate cancer: a randomised, double-blind study. Lancet 2011;377(9768):813-22.

5. Lipton A, Fizazi K, Stopeck AT, Henry DH, Brown JE, Yardley $D A$, et al. Superiority of denosumab to zoledronic acid for prevention of skeletal-related events: a combined analysis of 3 pivotal, randomised, phase 3 trials. Eur $\mathrm{J}$ Cancer 2012;48(16):3082-92.

6. Sutton RA, Mumm S, Coburn SP, Ericson KL, Whyte MP. "Atypical femoral fractures" during bisphosphonate exposure in adult hypophosphatasia. J Bone Miner Res 2012;27(5):987-94.

7. Bedogni A, Saia G, Bettini G, Tronchet A, Totola A, Bedogni $G$, et al. Osteomalacia: the missing link in the pathogenesis of bisphosphonate-related osteonecrosis of the jaw? Oncologist 2012;17(8):1114-9. 